\title{
Reprint
}

\section{Direction Finding in IEEE802.11 Wireless Networks}

A. Kalis and T. Antonakopoulos

\section{IEEE Transactions on Measurements and Instrumentation}

Vol. 51 No. 5, OCTOBER 2002, pp. 940-948

Copyright Notice: This material is presented to ensure timely dissemination of scholarly and technical work. Copyright and all rights therein are retained by authors or by other copyright holders. All persons copying this information are expected to adhere to the terms and constraints invoked by each author's copyright. In most cases, these works may not be reposted or mass reproduced without the explicit permission of the copyright holder. 


\title{
Direction Finding in IEEE802.11 Wireless Networks
}

\author{
Antonis Kalis, Member, IEEE, and Theodore Antonakopoulos, Senior Member, IEEE
}

\begin{abstract}
A novel direction-finding method for stations of IEEE802.11 wireless local area networks is presented in this paper. The method uses a switched beam array for determining the direction of arrival of the incident electromagnetic field in a time efficient way and associates certain medium access control MAC layer functions with different radiation patterns of the switched antenna array, in order to determine the proper orientation of directional beams on both entities of a communication link. The application of the proposed method to an IEEE802.11 wireless network is presented and it is depicted how the method improves the network performance without requiring any modifications to the existing MAC protocol.
\end{abstract}

Index Terms-Direction of arrival (DoA) estimation, directive antennas, IEEE80211, indoor radio communications, wireless LAN.

\section{INTRODUCTION}

H OME networking, mobile computing, and small-office/home (SOHO) multimedia are technological areas with a fast rising demand for extended wireless bandwidth. The wireless channel imposes several restrictions to this demand, due to the effect of multipath fading. One of the most efficient ways to confront the effect of multipath is by using directive antennas on both sides of the wireless link [1], [2]. A directive antenna on the receiver side attenuates the multipath instances arriving from different directions and that results in a stronger useful signal [e.g., the signal that comes directly from the transmitter's line of sight (LOS)]. Moreover, by using a directional antenna on the transmitter, most of the electromagnetic energy of the transmitted signal is directed to the receiver's direction; thus, the spurious reflections and diffractions of the signal, that cause multipath fading, are also minimized.

Optimum transmission performance over a wireless channel is achieved when both communicating devices can determine the relative direction of each other and turn their directional beam antennas accordingly. In a static network, where all stations have a fixed position, this can be planned beforehand and established permanently. In wireless data networks, the stations mobility increases the complexity of the network, since moving stations should constantly acquire and retain relative direction information on all other mobile stations. In mobile applications that have continuous data exchange in both directions, such as voice communication in cell phone applications, relative direction information may be acquired continuously and constantly by integrating a radio direction finding (RDF) algorithm in the

Manuscript received May 29, 2001; revised August 7, 2002. This work was supported in part by ATMEL SA in the framework of Project Aliakmon.

The authors are with the Department of Electrical Engineering and Computers Technology, University of Patras, Rio-Patras, Greece (e-mail: antonako@ ee.upatras.gr).

Digital Object Identifier 10.1109/TIM.2002.807806 physical layer, with the use of phased arrays or switched beam arrays [3]. In mobile computing networks, there is no continuously established link between transmitter and receiver and relative direction information is hard to acquire and retain. While a receiving station can determine the relative direction of the transmitting station, the transmitter is usually unable to perform the same procedure during transmission, since the flow of information is neither continuous nor full duplex. In such networks, pure physical layer functions that determine the direction of arrival (DoA) of the incoming signals are not adequate for determining the correct orientation of the antenna beams on both sides of a communication link. A straightforward way to achieve that is to force the network stations to exchange relative position information, but in this case the medium access control (MAC) layer should be altered and the solution would require a significant amount of network bandwidth.

The IEEE802.11 standards [4], [5] define the most popular technology for wireless LANs today. The technology is based on a number of physical layer protocols and a MAC layer. The physical layer of IEEE802.11 networks at $2.4 \mathrm{GHz}$ uses two modulation schemes: DBPSK for the preamble of each frame and DQPSK for MAC layer-related information, while in the 5-GHz band, orthogonal frequency division multiplexing (OFDM) is used and a number of sub-carriers are modulated using BPSK, QPSK, 16-QAM, and 64-QAM. The mobile stations establish peer-to-peer communications and exchange data asynchronously by implementing the distributed coordination function (DCF).

In this paper, we propose a method that uses physical and MAC layer functions for acquiring relative direction information on both sides of a packet switched communication link. On the receiver's side the proposed method is based on the RDF algorithm, which is related to the switched beam array characteristics. The switched arrays are preferred for mobile computing network applications compared to phased arrays, since they satisfy the cost, size, and power consumption restrictions of mobile terminals. The RDF algorithms for switched beam arrays have already been reported in the literature [6], but they target cell phone applications with less strict timing requirements than packet switching networks. A modified version of such an algorithm is adopted and integrated in the proposed direction finding method, in order to meet the MAC protocol timing restrictions. On the other hand, in order to update a transmitting station with new information regarding the relative direction of its receiver, the method associates some MAC layer frames with certain radiation patterns of the array, without altering the MAC protocol functionality. The proposed method focuses on the CSMA/CA protocol and its development was based on the IEEE802.11 characteristics, but is far more generally applicable. 


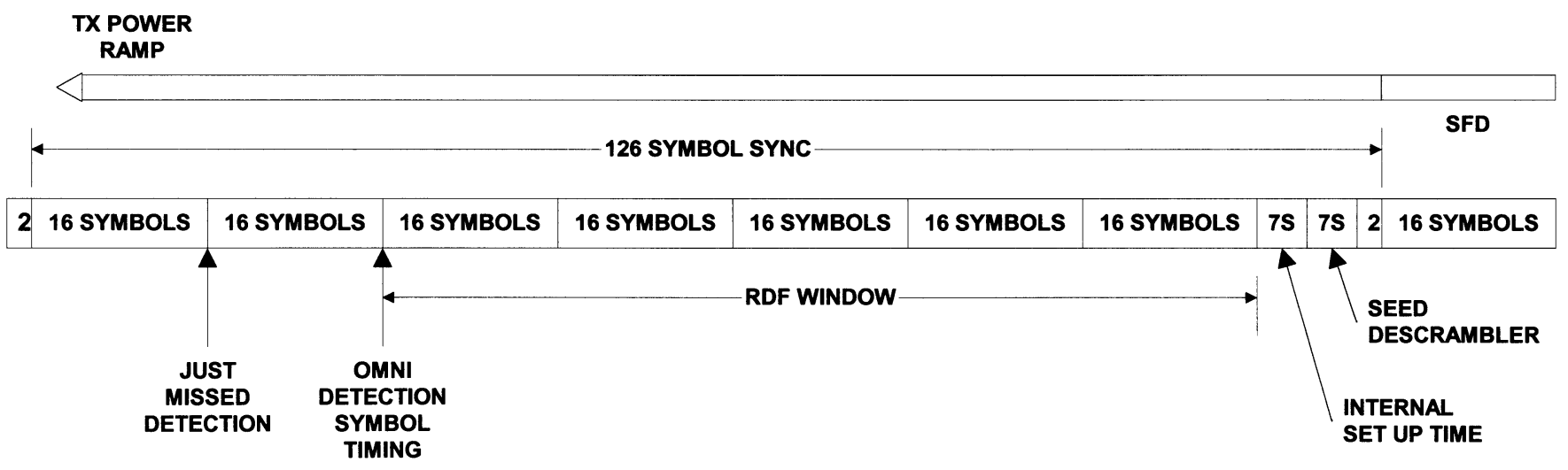

Fig. 1. Preamble of an IEEE802.11 DSSS physical header.

Section II describes the implemented direction finding method, which is based on switched beam arrays. Such a switched beam array, suitable for the implementation of the DoA method, is also presented. Section III describes the relative direction determination method in an IEEE802.11 wireless network, without requiring any modifications on the MAC layer functionality. Section IV presents various simulation results, which show how the proposed method improves the overall system performance.

\section{SwITCHED BEAM ARRAYS AND DiRECTION OF ARRIVAL}

In packet switching networks, there is no continuous flow of information between transmitting and receiving stations and the network traffic is characterized as bursty. Information is exchanged using frames that generally consist of four main fields: preamble, header, payload, and trailer. The preamble is a sequence of bits carrying information about the transmitter's timing and is used by the receiver in order to synchronize its timing circuits. The header includes information that is essential to the functionality of the wireless network protocol (e.g., destination and source MAC addresses), while the payload contains the information that has to be transferred from one station to the other. Finally, the MAC trailer is used for detecting any transmission errors. In such a mobile network, the antenna steering must be performed during the transmission of the preamble, since erroneous reception of any header bits will result in the rejection of the whole frame.

Since the motivation of this work was to improve the performance of IEEE802.11 WLANs, in the rest of this paper, we will use the restrictions imposed by the IEEE802.11 protocol specifications. The standard frame of the IEEE802.11 protocol [4] supports a 128-symbos preamble period for each transmitted frame, which results in $128 \mu \mathrm{s}$ of preamble time at $1 \mathrm{Mbps}$. According to the IEEE802.11b standard [5] the 128-symbols preamble is the mandatory long preamble, which allows interoperation with the current $1 \mathrm{Mbps}$ and $2 \mathrm{Mbps}$ DSSS specification, while an optional short preamble has been defined which has 56 symbols duration and the same baud rate. In some long preamble IEEE802.11 implementations, the strength of the incoming signal is calculated every $16 \mu$ s by using the received signal strength indication (RSSI) signal of the baseband con- troller, although other implementations with shorter RSSI calculation times are possible. Fig. 1 shows that at this sampling rate and in order to extract the synchronization information at the receiver's circuits, the maximum number of RSSI samples, which are available for finding the direction of arrival of the incoming signal, is five. This number corresponds to $80 \mu \mathrm{s}$, which determine the maximum duration for the convergence of the direction finding algorithm. This time window corresponds to the worst-case scenario, where the receiver does not detect an incoming signal during the first sample of RSSI; it needs one sampling period in order to synchronize the internal clock for the RDF process and needs one more sampling period for setting up the start of frame delimiter (SFD) detection.

\section{A. Direction of Arrival Techniques}

There are many proposals in the literature discussing how the directional beam of a mobile terminal, can be used to minimize the effect of multipath [6]-[8]. Most of the proposed algorithms are targeting cell phone applications and they use phased arrays in the base station. These algorithms are inadequate for mobile computing applications, since they assume that there is a continuous flow of data in both directions of the communication link and moreover they are based on phased arrays, which are not easily integrated in a mobile station, due to their cost and size.

Switched antenna arrays are more suitable for wireless LANs due to their reduced complexity. Switched arrays and antenna diversity schemes have already been integrated in wireless networks [9] and signal evaluation algorithms that determine the performance of these algorithms have been widely reported. Preston et al. in [6] have proposed a very interesting method for acquiring DoA information using a switched beam array. DoA finding methods are based on the following concept: the switched beam array produces $\mathrm{N}$ different radiation patterns, which divide the azimuth plane in $\mathrm{N}$ equal angular sectors. In order to find the DoA, the receiver scans all these patterns and estimates the power of the received signal by measuring the RSSI signal in each sector. By comparing the different RSSI values, the receiver can determine the relative angle of arrival of the incident signal with varying accuracy, depending on the comparison method. Due to the fact that the computational burden of these methods is minimal, the time needed to compute the relative DoA is proportional to the total number of the $N$ different array sectors. 


\section{B. Switched Beam Array}

The method proposed in this paper uses a switched beam array that produces omnidirectional and directional beams. Such arrays have been recently reported in the literature and are categorized either as patch [10], slot [11], or monopole arrays. In [11], we have proposed an active switched printed slot antenna array for the 5.2-GHz band that generates an omnidirectional pattern and many directional patterns in the azimuth plane, the narrower of which has a directivity of $8.56 \mathrm{dBi}$. Although this array was developed for the 5.2-GHz frequency band, a new version, suitable for the $2.4-\mathrm{GHz}$ ISM frequency band of IEEE802.11, has been developed, having similar radiation patterns as those reported in [11]. Fig. 2 shows all the different radiation patterns that are adequate to cover all the azimuth angles by driving a different number of elements. This array either generates an omnidirectional radiation pattern, or divides the azimuth plane in a number of equal angular sectors. There are two types of angular sectors, the wide angle and the narrow angle sectors. In our design, we have defined $180^{\circ}$ wide-angle sectors and $60^{\circ}$ narrow-angle sectors. Therefore, the azimuth plane is divided into $N_{1}=2$ wide-angle sectors and each wide-angle sector is further divided into $N_{2}=3$ narrow-angle sectors. These radiation patterns have a crucial effect on the implementation of the DoA finding method, as it is described in the next section.

In order to integrate such a switched array in a WLAN implementation, a feeding network that divides/combines the RF power to/from a variable number of outputs, in order to produce variable diversity gain beams, with acceptable circuit losses has been developed and its basic concept has been presented in [12]. This feeding-network building block is based on a modified Wilkinson power divider/combiner circuit, uses high-speed $p-i$ - $n$ diodes and can be used either in stand-alone or cascaded configurations, in order to produce the driving signals of all radiation patterns.

\section{Direction Finding Method}

We consider a switched beam array that can produce the following radiation patterns.

- An omnidirectional pattern.

- $N_{1}$ wide-angle sectors of $360 / N_{1}{ }^{\circ}$ each.

- $N_{1} N_{2}$ narrow angle sectors of $360 / N_{1} N_{2}{ }^{\circ}$ each. $N_{2}$ narrow-angle radiation patterns cover more efficiently the same angle as a single wide-angle radiation pattern.

- In general, if a sector is further divided into $N_{k}$ narrower sectors, then the radiation angle of these sectors is $360 / \prod_{i=1}^{k} N_{i}{ }^{\circ}$.

When the receiver is in idle mode, the antenna is switched to the omnidirectional pattern. Once the RSSI signal exceeds a certain threshold level, meaning that the antenna has received an incident signal, the procedure for determining the transmitter's relative direction is activated. The receiver initially measures the RSSI of all $N_{1}$ sectors. Once the sector with the best signal is determined, then the receiver repeats the same procedure for all $N_{2}$ narrower sectors of this sector. This tree-like search algorithm continues until the $N_{k}$ sector with the best performance is determined and the DoA finding method's accuracy is equal to the beam-width of
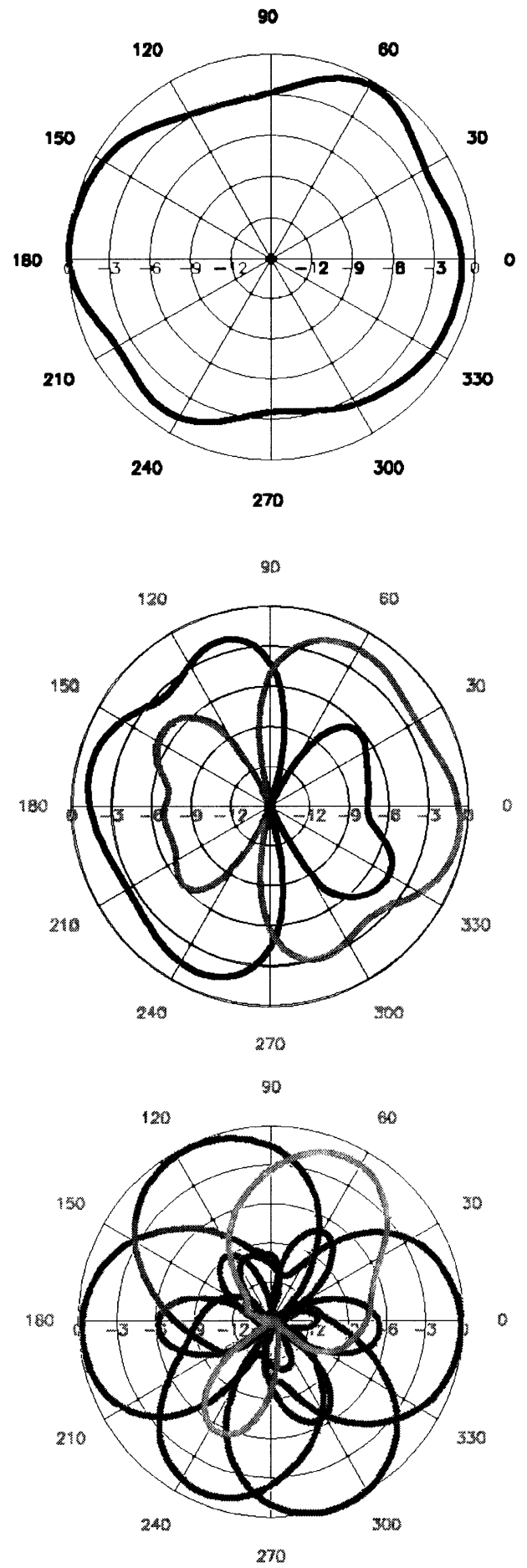

Fig. 2. Different switched array radiation patterns.

the narrowest sector. The first step is used for performing coarse angular location of the transmitting station, while the next steps are performed for more precise angular location. Fig. 3 shows how the algorithm envolves in a switched array that has radiation patterns of $90^{\circ}$ and $45^{\circ}$. In idle mode, the array operates in the omnidirectional configuration, but when transmission has been detected, the array initially uses four $90^{\circ}$ radiation patterns for a first estimate of the direction of arrival and then uses the two $45^{\circ}$ radiation patterns that determine the most accurate estimation of DoA. Following this procedure, the proposed RDF algorithm 


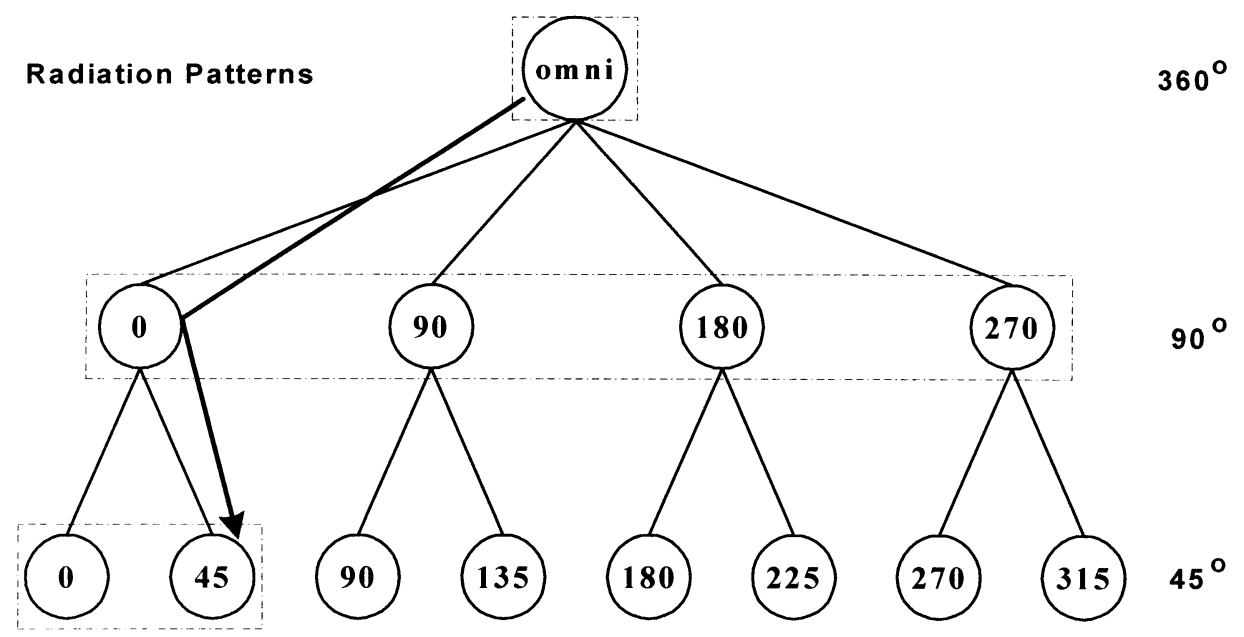

Fig. 3. Tree-like search algorithm for beamwidths of $90^{\circ}$ and $45^{\circ}$.

always finds the sector that receives the strongest signal, regardless of the number and strength of the multi-paths received by the antenna. The total time required in order to estimate the best direction of arrival is proportional to the sum: $N=\sum_{i=1}^{k} N_{i}$ and not to the total number of narrow sectors: $N=\prod_{i=1}^{k} N_{i}$. It is evident that the proposed method converges fast to the best direction of arrival and as it is explained in the following example, it is suitable for IEEE802.11 wireless networks. We consider the above described method and the switched beam array with the radiation patterns of Fig. 2, where $N_{1}=2$ and $N_{2}=3$. The proposed direction of arrival finding technique needs five samples $(80 \mu \mathrm{s})$ to determine the DoA of the incoming signal with an accuracy of $60^{\circ}$, as opposed to the six samples $(96 \mu \mathrm{s})$ needed by the algorithm based on exhaustive testing of the narrowest radiation patterns.

\section{DIRECTION FINDING}

In the previous section, we described a method for determining the DoA of an incoming signal, so that a station receiving a data frame can switch to a directional beam pointing at the transmitter's LOS. In order to minimize the strong multipaths that arrive at the receiver within a small angular distance from the LOS, it is equally crucial to use a directional beam also at the transmitter [2]. This can be achieved by using the previously mentioned DoA method and by associating certain MAC layer functions with different radiation patterns of the transmitter's antenna. The method is based on the development of a beam management function that communicates with the physical and the MAC layer entities and remains transparent to the functionality of these two layers. The MAC layer of IEEE802.11 wireless networks uses the CSMA/CA access protocol and the proposed method can be applied to any wireless network that uses the same or similar access mechanism.

\section{A. Medium Access Protocol}

The CSMA/CA protocol confronts the problem of collisions due to "hidden terminals" in wireless networks by using an exchange of short packets before the actual data transmission. For example, as it is shown in Fig. 4 where stations A, B, and C belong to the same WLAN, station C may not sense that station B is transmitting a packet (e.g., to station A) and it may also send a packet, causing a collision at station $\mathrm{A}$. In order to reduce the collision probability, the CSMA/CA protocol defines the Virtual Carrier Sense mechanism.

According to this mechanism, a station having to transmit a user packet initially transmits a short control frame called request-to-send (RTS), which includes the source address, destination address and the estimated duration of data transaction. The receiving station, whose MAC address is the same as the destination address of the RTS, answers with a response control frame called clear-to-send (CTS), which includes the same duration information. All stations receiving either the RTS and/or the CTS frame set their virtual carrier sense indicator [called network allocation vector (NAV)] to an appropriate value and remain idle for a given duration of time. This mechanism reduces the collision probability due to a station that is "hidden" from the data exchange initiator, since the "hidden" station senses the CTS frame transmission and considers the medium busy, until the end of the transaction, as defined in the content of CTS, although it did not receive the initial RTS frame and it will not receive the actual data frame. The transmission time information contained in the RTS/CTS frames protects the network from collisions during the transmission of the acknowledgment frame (ACK). Fig. 4 also shows a data transaction between stations A and $\mathrm{B}$, the transmitted frames and the NAV parameter settings on neighbor stations. The exchange of these control frames can be exploited in order to determine the relative direction of the communicating stations, without affecting the CSMA/CA protocol functionality.

\section{B. Beam Management Function}

The proposed direction finding method exhibits its best performance in a slow-time-varying environment, with a dominant LOS signal. The characteristics of the indoor channel, which are thoroughly described in [13], meet this specification. In such an environment, the channel characteristics do not change significantly over a period of some milliseconds, which corresponds to the duration of a packet exchange. Fig. 5 shows an example 


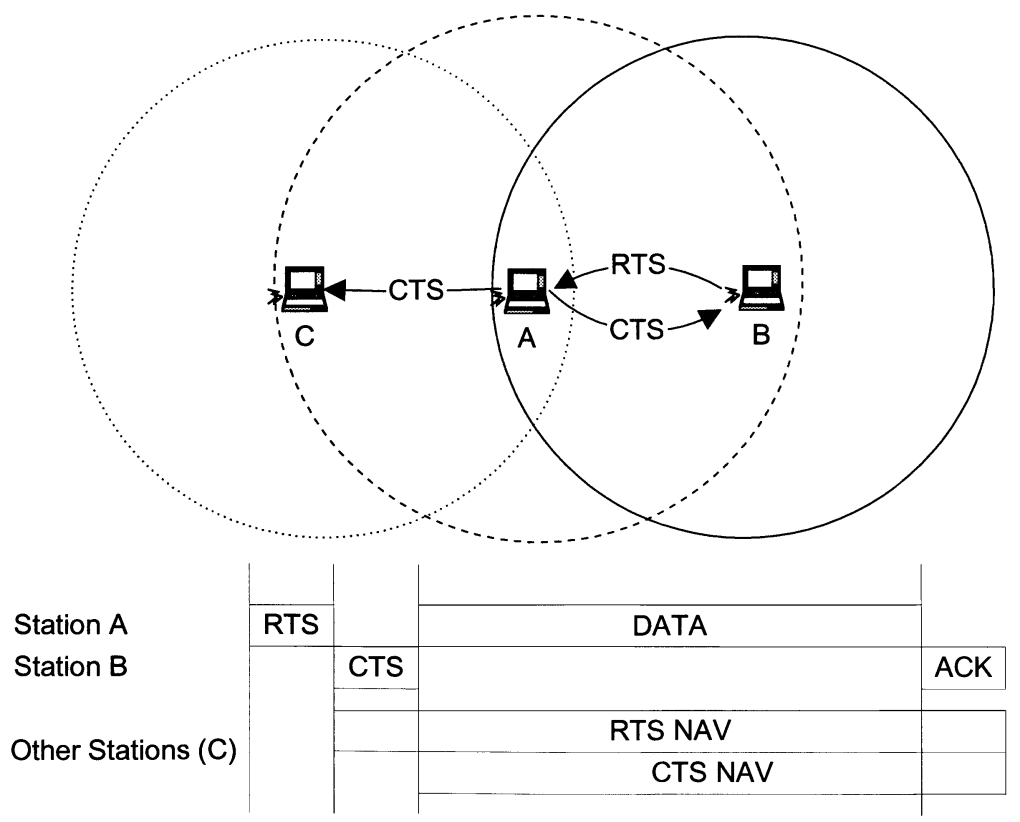

Fig. 4. Collision avoidance algorithm in IEEE802.11.

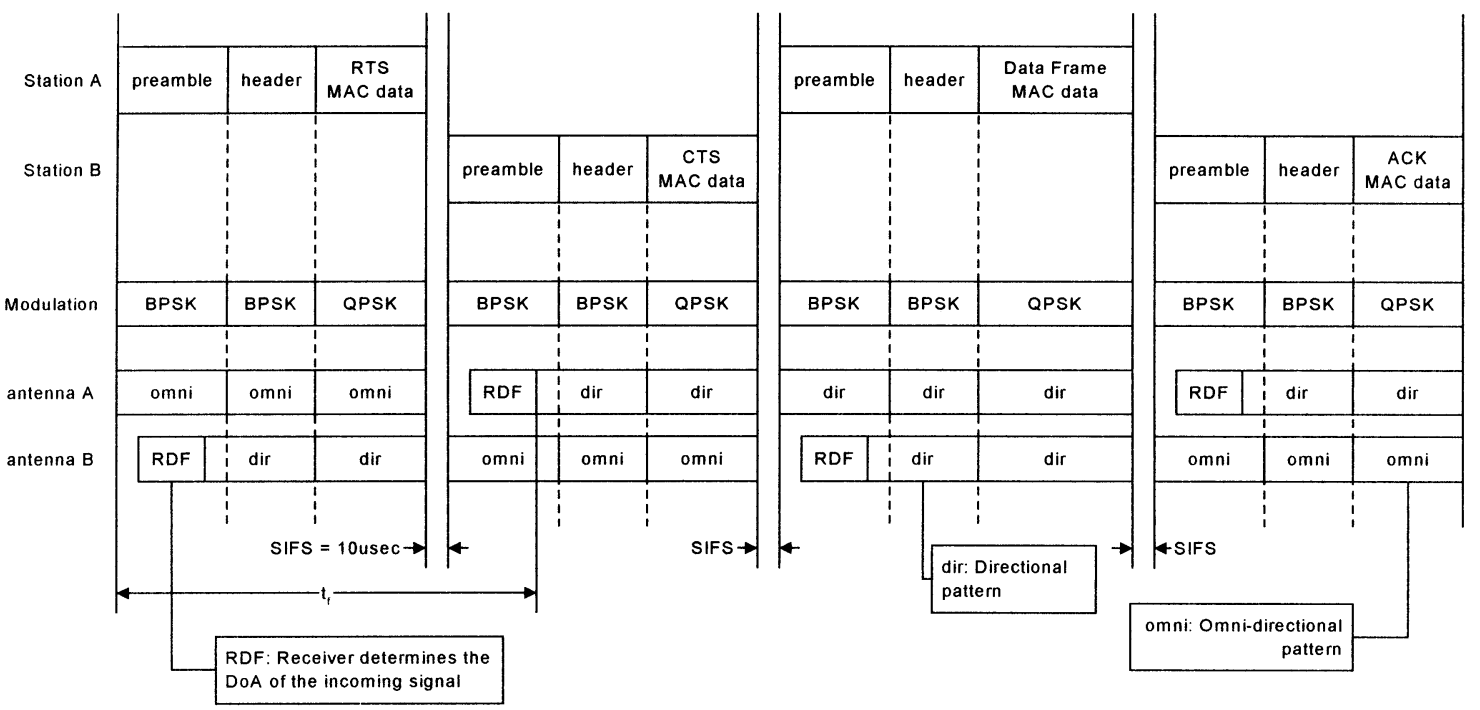

Fig. 5. Direction finding method during data exchange.

of using the proposed method for obtaining relative direction information on both sides of the communication link. This figure gives details on the radiation patterns used on both communicating stations and the modulation schemes used. Station A has to transmit a frame to station B and uses the omnidirectional pattern of its switched antenna array in order to send the RTS frame. All stations that "hear" the transmission will initially receive the physical layer header of the packet. During the reception of the header's preamble, the DoA is determined, using the direction finding method described in the previous section. At the end of the RTS frame transmission, station B sends a CTS frame back to station A. During the reception of the preamble of this frame, station A determines the relative direction of station B. At the end of RTS and CTS frames, all network contentions have been resolved and the stations are free to use their directional beams for data transmission. Assuming that the indoor channel does not change significantly over the time of a CTS + DATA frame transmission sequence, station A uses the directional beam that was chosen during the preamble period of the CTS frame and transmits the data frame. Station B uses the same direction finding process and at the end of the data frame preamble, both stations know the relative direction of each other and direct their antenna beams accordingly. During the ACK frame transmission, station A may refresh its direction information for station B.

Using the previously described direction finding method, the time $t_{f}$ required for station A to acquire relative direction information for station B is $394 \mu \mathrm{s}$, assuming that the 2 Mbps DSSS version of IEEE802.11 is used. If 5.5 or $11 \mathrm{Mbps}$ are used for MAC payload transmission, as in IEEE802.11b, this time becomes even shorter. The relative direction information is not refreshed during the transmission of the data frame. During this 
TABLE I

RMS DELAy SPREAD For DifFERENT ANTENNA BEAMWIDTHS (RMS DElay SpREAD IN NANOSECONDS; IMPROVED SNR IN DECIBALS)

\begin{tabular}{|c|c|c|c|c|c|c|c|c|c|c|}
\hline & \multicolumn{5}{|c|}{ Clusters: 5 Rays/cluster $=4$} & \multicolumn{5}{|c|}{ Clusters: $15 \quad$ Rays/cluster $=10$} \\
\hline Angle $\left(^{\circ}\right)$ & $\begin{array}{c}\text { OO } \\
\text { rmsDS }\end{array}$ & $\begin{array}{c}\text { OD } \\
\text { rmsDS }\end{array}$ & $\begin{array}{c}\text { DD } \\
\text { rmsDS }\end{array}$ & $\begin{array}{c}\text { OO-OD } \\
\text { imprSNR }\end{array}$ & $\begin{array}{c}\text { OO-DD } \\
\text { imprSNR }\end{array}$ & $\begin{array}{c}\text { OO } \\
\text { rmsDS }\end{array}$ & $\begin{array}{c}\text { OD } \\
\text { rmsDS }\end{array}$ & $\begin{array}{c}\text { DD } \\
\text { rmsDS }\end{array}$ & $\begin{array}{c}\text { OO-OD } \\
\text { imprSNR }\end{array}$ & $\begin{array}{c}\text { OO-DD } \\
\text { imprSNR }\end{array}$ \\
\hline 330 & 50.01 & 49.99 & 49.80 & 0.94 & 1.70 & 49.90 & 49.83 & 49.71 & 0.92 & 1.65 \\
\hline 300 & 49.88 & 49.69 & 49.29 & 1.16 & 2.05 & 49.75 & 49.65 & 49.49 & 1.15 & 2.00 \\
\hline 270 & 50.54 & 50.10 & 49.47 & 1.74 & 2.69 & 49.69 & 49.41 & 49.16 & 1.68 & 2.55 \\
\hline 240 & 50.31 & 49.35 & 48.56 & 2.29 & 3.27 & 49.53 & 49.16 & 48.89 & 2.19 & 3.07 \\
\hline 210 & 49.86 & 47.85 & 46.79 & 3.03 & 4.04 & 49.87 & 49.14 & 48.81 & 2.85 & 3.75 \\
\hline 180 & 50.37 & 47.23 & 45.58 & 3.92 & 4.97 & 49.71 & 48.62 & 48.24 & 3.51 & 4.41 \\
\hline 150 & 50.31 & 45.77 & 43.38 & 5.04 & 6.29 & 49.77 & 48.29 & 47.68 & 4.43 & 5.36 \\
\hline 120 & 50.10 & 43.22 & 39.75 & 6.11 & 7.51 & 49.92 & 47.59 & 46.62 & 5.49 & 6.47 \\
\hline 90 & 50.04 & 42.52 & 36.89 & 7.96 & 10.01 & 49.89 & 47.59 & 44.55 & 6.90 & 7.97 \\
\hline 60 & 49.96 & 40.77 & 33.22 & 10.19 & 13.52 & 49.65 & 44.63 & 41.47 & 8.96 & 10.37 \\
\hline 30 & 50.09 & 37.73 & 26.89 & 15.03 & 21.39 & 49.74 & 40.90 & 34.23 & 13.14 & 15.64 \\
\hline
\end{tabular}

period, the relative locations of both stations do not change significantly and the accuracy of the relative direction information does not deteriorate.

The proposed method can also be applied when only one of the communicating devices uses directional antennas, since each station behaves independently from the other and does not set any requirements on the other station's capability to use the same or another type of directive antenna.

\section{PeRformance AnAlysis}

The use of directional antennas is a form of antenna diversity that can be used for increasing the data rate in wireless networks. Directional antennas transmit more power to a specific direction, compared to an omnidirectional antenna with the same input power, but their major advantage is that they improve the quality of the received signal by reducing the multipath effects. It has been demonstrated in [1] that $60^{\circ}$ directional antennas enhance the received signal quality significantly and reliable high-speed data transmissions can be achieved in the indoor environment; while in [14] simulation results show that a single $60^{\circ}$ beam-width antenna reduces the delay spread by more than $20 \%$.

In order to evaluate the performance of the proposed direction finding method, we have to analyze how the radio channel is affected when directional antennas are used, by estimating the value of rms delay spread, since it determines the performance degradation caused by intersymbol interference [15], [16]. The channel performance was studied for all possible combinations of transmitting and receiving antenna beams. Due to the bi-directionality of the wireless channel, the following cases were considered:

- omnidirectional to omnidirectional (OO)

- omnidirectional to directional (OD)

- directional to directional (DD) where the OO case is used as the reference for the other two cases. In the OD case, one of the communicating stations uses the proposed method, while the other one behaves as a wireless station without any antenna directivity. When both stations have directive antennas and use the proposed method, the DD case applies.

In order to evaluate the performance of the proposed method, the statistical model described in [17] has been used. This model is an extension of the time-only model presented in [13], provides temporal and spatial information for the indoor multipath channel, uses probability distributions for estimating the channel's behavior and allows the inclusion of directive antennas. We consider that arrivals come in attenuated clusters in time and the rays within a cluster also decay with time. Both decaying patterns are exponential in time and are characterized by the cluster arrival decay time constant and the ray arrival decay time constant. We consider clusters of arrivals in angle following the Laplacian distribution. In this model, the temporal and spatial channel parameters are considered independent of one another. In our simulations, we assumed that the main lobe of each directive antenna points directly to the other station, which is valid when the proposed direction finding method is used. We also assumed that the channel could be considered reciprocal, meaning that the transmitter and receiver designations can be exchanged.

We consider a wireless network having a 50-ns delay spread when omnidirectional antennas are used in both communicating devices. The rms delay spread $\tau_{r m s}$ is calculated using the following equation:

$$
\tau_{r m s}=\sqrt{\frac{\sum\left(t_{i}-\tau_{m}-\tau_{0}\right)^{2} a_{i}^{2}}{\sum a_{i}^{2}}}
$$




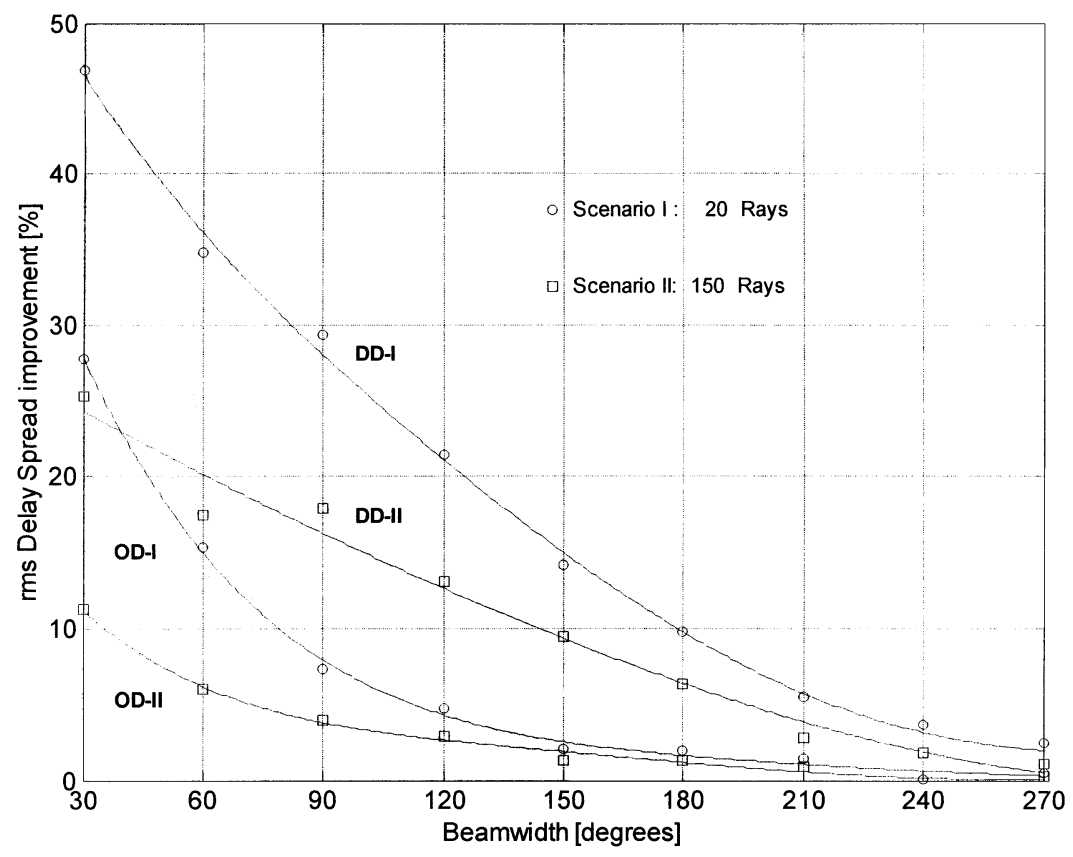

Fig. 6. RMS delay spread improvement versus beamwidth.

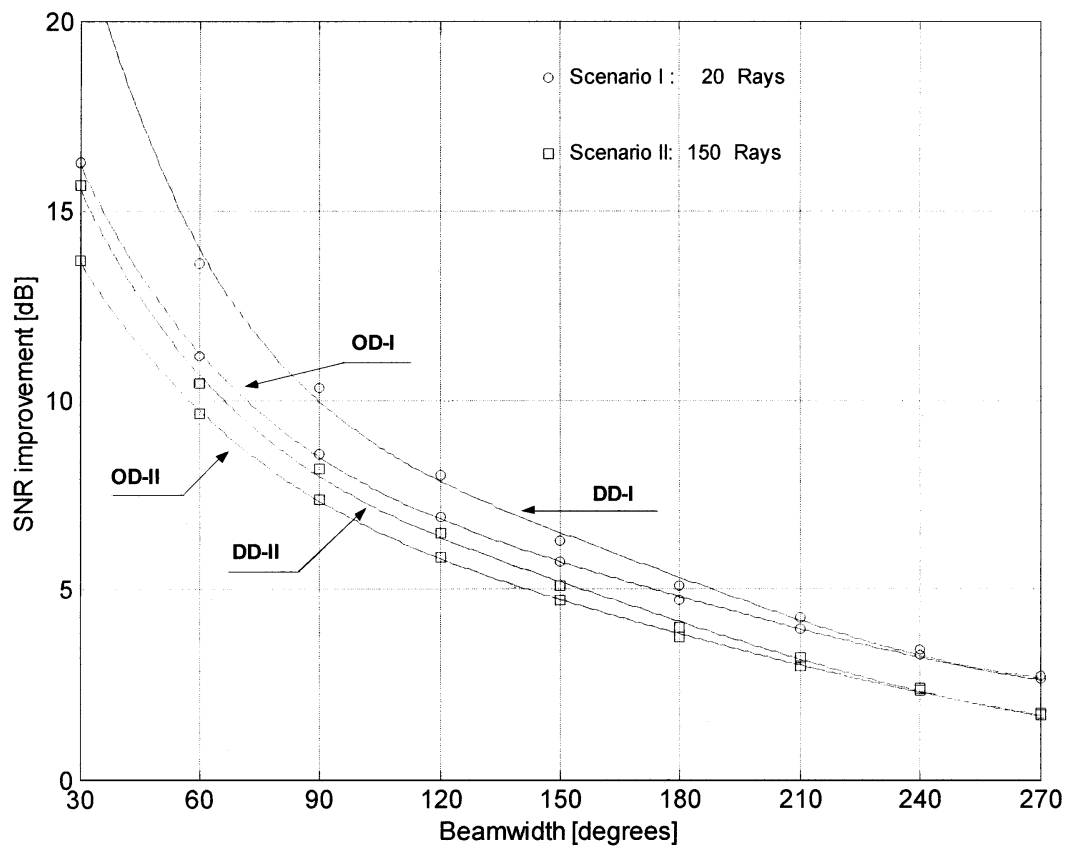

Fig. 7. SNR improvement versus beamwidth.

where $\tau_{0}$ is the arrival time of the first arriving path, $t_{i}$ is the arrival time of the $i$-multipath component, $a_{i}$ is its amplitude, and $\tau_{m}$ is the mean excess delay, given by

$$
\tau_{m}=\frac{\sum\left(t_{i}-\tau_{0}\right) a_{i}^{2}}{\sum a_{i}^{2}}
$$

Table I shows the rms delay spread for different beam-widths and the respective SNR improvement compared to the OO case, based on simulation measurements. Figs. 6 and 7 show how the use of directional antennas in one or both communicating devices can decrease the rms delay spread and improve the channel SNR respectively. In these figures, two different scenarios are shown. Scenario I uses a small number of clusters (5) and a small number of rays per cluster (4), resulting in a total of 20 multipath components, in addition to the LOS signal, while Scenario II is based on more clusters (15) and more rays per cluster (10), resulting in a total of 150 multipath components.

Using the $60^{\circ}$ directional antennas described in Section II, the rms delay spread of Scenario I is improved by $18 \%$ in the OD case and 35\% in the DD case, while in Scenario II the improvement is $11 \%$ and $17 \%$ respectively. Less rms delay spread means better transmission conditions. In [18], the bit error probability of DBPSK has been associated with the rms delay spread. If $T_{b}$ is the bit length, for small delay spreads, optimum fixed 


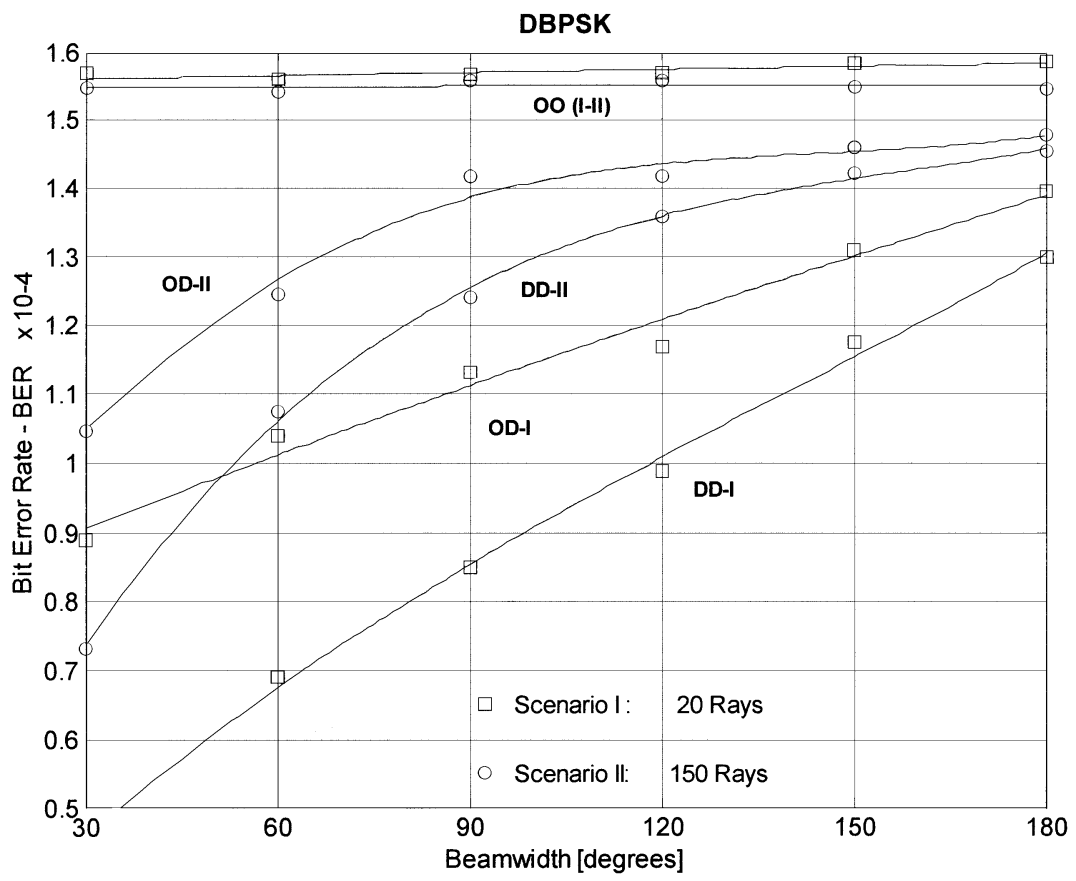

Fig. 8. DBPSK bit error rate versus beamwidth.

sampling times and amplitudes following the Rayleigh distribution, the BER of DBPSK is given by $\left(\tau_{r m s} / 4 T_{b}\right)^{2}$. This can be considered as the lower limit of BER, since in our simulations a dominant LOS signal is always present, resulting in received signals of better quality. Fig. 8 shows how the worst case bit error rate is associated to the beam-width of the antennas used in the wireless stations and Rayleigh multipath conditions. Using directional antennas of $60^{\circ}$, the bit error rate decreases by more than $40 \%$, while the rms delay spread decreases by more than $20 \%$. Directional antennas with beam-width larger than $120^{\circ}$ have negligible effect on the channel bit error rate. The channel SNR is also improved when directional antennas are used, as it is shown in Fig. 7 for the two scenarios investigated in this work. The $60^{\circ}$ directional antennas increase the channel's SNR by $8-10 \mathrm{~dB}$ in the OD case and $11-14 \mathrm{~dB}$ in the DD case.

\section{CONCLUSION}

In this paper, we presented a direction finding method for IEEE802.11 wireless LANs. The method is based on the use of switched beam arrays with variable diversity gain and an efficient direction finding algorithm. The proposed method exploits the RTS and CTS frames used in IEEE802.11 in order to acquire direction information on both sides of the communication link, without affecting the MAC layer functionality and without requiring any additional bandwidth. As the simulation results show, the performance of the proposed method depends on the channel conditions (number of multipath components, amplitude, time and angle of arrival distributions), but in all cases, the proposed method decreases the channel's delay spread and improves its bit error rate.

\section{REFERENCES}

[1] T. A. Freeburg, "Enabling technologies for in-building network communications-four technical challenges and four solutions," IEEE Trans. Veh. Technol., vol. 29, pp. 58-64, Aug. 1991.

[2] P. Driessen, "Gigabit/s indoor wireless systems with directional antennas," IEEE Trans. Commun., vol. 44, pp. 1034-1043, Aug. 1996.

[3] P. Lehne and M. Pettersen, "An overview of smart antenna technology for mobile communications systems," IEEE Commun. Surv, vol. 2, Aug. 1999.

[4] Part 11: Wireless LAN Medium Access (MAC) and Physical Layer (PHY) Specifications., IEEE Std. 802.11-1997, June 1997.

[5] Part 11: Wireless LAN Medium Access Control (MAC) and Physical Layer (PHY) specifications: Higher-Speed Physical Layer Extension in the 2.4 GHz Band., IEEE Std. 802.11b-1999, Sept. 1999.

[6] S. Preston, V. Thiel, T. Smith, S. O'Keefe, and J. Lu, "Base-station tracking in mobile communications using a switched parasitic antenna array," IEEE Trans. Antennas Propagat., vol. 46, no. 6, pp. 841-844, June 1998.

[7] L. Jianxia, J. Zeidler, and S. McLaughlin, "Bit-error probability analysis of compact antenna arrays with maximal-ratio combining in correlated nakagami fading," in IEEE Workshop Sens. Array Multichannel Signal Processing, 2000, pp. 52-57.

[8] G. Yang, K. Pahlavan, and T. J. Holt, "Sector antenna and DFE modems for high speed indoor radio communications," IEEE Trans. Veh. Technol., vol. 43, pp. 925-933, Nov. 1994.

[9] PRISM $^{\circledR}$ Chipset [Online]. Available: http://www.intersil.com

[10] H. Iwasaki, "Slot coupled back-to-back microstrip antenna with an omni or a bi-directional radiation pattern," Proc. Inst. Elect. Eng.-Microwave. Antennas Propagat., vol. 46, pp. 219-223, June 1999.

[11] A. Kalis, Th. Antonakopoulos, and V. Makios, "A printed circuit switched array antenna for indoor communications," IEEE Trans. Consumer Electron., vol. 46, pp. 531-538, Aug. 2000.

[12] _ " "A power divider/combiner block for switched beam arrays," in IEEE Int. Symp. Ind. Electron., ISIE, L'Aquila, Italy, July 2002.

[13] A. Saleh and R. Valenzuela, "A statistical model for indoor multipath propagation," IEEE J. Selected Areas Commun., vol. SAC-5, pp. 128-137, Feb. 1987.

[14] M. Lu, T. Lo, and J. Litva, "A physical spatio-temporal model of multipath propagation channels," in Proc. 47th IEEE Veh. Technol. Conf., vol. 2, 1997, pp. 810-814.

[15] H. Hashemi, "The indoor radio propagation channel," Proc. IEEE, vol. 81, pp. 943-968, July 1993.

[16] K. Pahlavan and A. H. Levesque, Wireless Information Networks. New York: Wiley, 1995. 
[17] Q. H. Spencer, B. D. Jeffs, M. A. Jensen, and A. L. Swindlehurst, "Modeling the statistical time and angle of arrival characteristics of an indoor multipath channel," IEEE J. Selected Areas Commun., vol. 18, pp. 347-459, Mar. 2000.

[18] A. F. Molisch, J. Fuhl, and E. Bonek, "Exact computation of the error floor of DBPSK in two-delay mobile radio channels," in Proc. Second ITG-Fachtagung Mobile Kommunikation, Neu-Ulm, Germany, Sept. 1995 , pp. 263-270

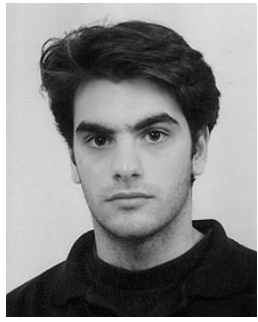

Antonis Kalis (M'01) was born in Athens, Greece, in 1974. He received the Electrical Engineering Diploma degree in 1997 from the Department of Electrical Engineering and Computer Technology, University of Patras, Patras, Greece.

In September 1997, he joined the Laboratory of Electromagnetics, University of Patras, participating in various $R \& D$ projects for the Greek Government and the European Union, as research staff. Today he works as a Research Engineer at the Research Unit 7 of the Computer Technology Institute.

Mr. Kalis is a member of the Technical Chamber of Greece.

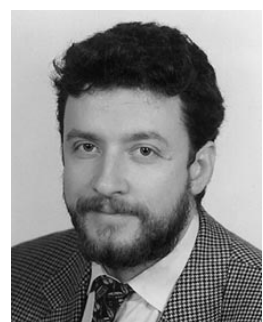

Theodore Antonakopoulos (M'88-SM'99) was born in Patras, Greece, in 1962. He received the Electrical Engineering Diploma degree and the Ph.D. degree from the Department of Electrical Engineering at the University of Patras in 1985 and 1989, respectively.

In September 1985, he joined the Laboratory of Electromagnetics, University of Patras, participating in various R\&D projects for the Greek Government and the European Union, initially as a research staff member and subsequently as the Senior Researcher of the Communications Group. Since 1991, he has been on the faculty of the Electrical Engineering Department, University of Patras, where he is currently an Associate Professor. His research interests are in the areas of data communication networks, LANs, and wireless networks, with emphasis on performance analysis, efficient hardware implementation, and rapid prototyping. He has more than 80 publications in the above areas and is actively participating in several R\&D projects of European industries.

Dr. Antonakopoulos serves in the Program Committee of the IEEE International Workshop on Rapid System Prototyping and is a member of the Technical Chamber of Greece. 